\title{
Inhibition of cell proliferation and radioresistance by miR- 383-5p through targeting RNA binding protein motif (RBM3) in nasopharyngeal carcinoma
}

\author{
Rui Ma ${ }^{1 \#}$, Peng Gao ${ }^{2 \#}$, Hua Yang ${ }^{1}$, Jing Hu$^{1}$, Jing-Jing Xiao $^{3}, \mathrm{Mei} \mathrm{Shi}^{1}, \mathrm{Li}^{-\mathrm{Na}} \mathrm{Zhao}^{1}$ \\ ${ }^{1}$ Department of Radiation Oncology, Xijing Hospital, Air Force Medical University, Xi'an, China; ${ }^{2}$ Department of Radiation Medicine, The Faculty \\ of Preventive Medicine, Air Force Medical University, Xi'an, China; ${ }^{3}$ Department of Thyroid-breast-vascular Surgery, Xijing Hospital, Air Force \\ Medical University, Xi'an, China \\ Contributions: (I) Conception and design: LN Zhao, M Shi, R Ma; (II) Administrative support: LN Zhao; (III) Provision of study materials or patients: \\ R Ma, P Gao; (IV) Collection and assembly of data: R Ma, P Gao, H Yang; (V) Data analysis and interpretation: R Ma, J Hu, J Xiao; (VI) Manuscript \\ writing: All authors; (VII) Final approval of manuscript: All authors. \\ \#These authors contributed equally to this work. \\ Correspondence to: Mei Shi; Li-Na Zhao. Department of Radiation Oncology, Xijing Hospital, Air Force Medical University, No. 127 West Changle \\ Road, Xi'an 710032, China. Email: mshi82@hotmail.com; zhaolinazln@outlook.com.
}

Background: RNA binding protein motif (RBM3) is associated with radioresistance in nasopharyngeal carcinoma (NPC), and miR-383-5p was predicted to target the 3'-untranslated region (3'UTR) of RBM3 messenger RNA (mRNA). Our study aimed to investigate the role and the mechanisms of miR-383-5p targeting RBM3 in NPC cell proliferation and radioresistance (RR).

Methods: The expression of miR-383-5p was detected by Real-time quantitative PCR (qRT-PCR) between RS (Radiosensitivity) and RR (Radioresistance) NPC patient- tissue specimens and cell lines. Cell Counting Kit-8 (CCK-8) and Clonogenic survival assay were applied to analyze the effect of miR-383-5p on NPC cell proliferation and radioresistance. Possible downstream target of miR-383-5p in NPC cells, RBM3was evaluated by luciferase assay and qRT-PCR. miR-383-5p inhibited NPC cell proliferation and radioresistance through RBM3 by rescue experiments. The effect of miR-383-5p on radiation-induced apoptosis was explored through Flow cytometric analysis and Western blotting. Western blotting was analyzed the molecular of RBM3-mediated Jun N-terminal kinase (JNK) and extracellular signal-related kinase (ERK) signaling pathways

Results: The expression of miR-383-5p was decreased in radioresistant NPC tissues and cells. miR-383$5 \mathrm{p}$ inhibited cell proliferation and radioresistance in CNE1/IR cells. We also observed that therapeutic administration of a miR-383-5p agomir dramatically sensitized NPC xenografts to radiation in a mouse model. Conversely, in the same xenograft model, administration of a miR-383-5p antagomir dramatically increased NPC resistance to radiation. miR-383-5p targeted the 3'UTR of RBM3. miR-383-5p inhibited NPC cell proliferation and radioresistance through RBM3. Finally, we found that miR-383-5p increased radiation-induced apoptosis, activated JNK signaling, and inhibited ERK signaling.

Conclusions: Our study revealed that miR-383-5p targeted the 3'UTR of RBM3 and contributed to the efficacy of NPC radiation therapy by altering the RBM3-mediated JNK and ERK signaling pathways.

Keywords: Apoptosis; extracellular signal-related kinase (ERK); Jun N-terminal kinase (JNK); miR-383-5p; nasopharyngeal carcinoma (NPC); radioresistance

Submitted Aug 19, 2020. Accepted for publication Jan 14, 2021.

doi: $10.21037 / \mathrm{atm}-20-6881$

View this article at: http://dx.doi.org/10.21037/atm-20-6881 


\section{Introduction}

Nasopharyngeal carcinoma (NPC) is one of the most common malignant head and neck tumors, and is especially prevalent in the southern region of China, the Southeast Asia (1). Radiotherapy is the primary treatment for NPC (1), because $90 \%$ of cases are poorly distinguished squamous cell carcinomas that exhibit radiosensitivity. However, radioresistance remains a significant obstacle for NPC treatment in a subset of patients (2). For this reason, radioresistance biomarkers are currently being investigated to enhance NPC treatment efficacy. We developed radioresistant NPC cell lines and showed that the RNA binding protein motif (RBM3) levels predict the NPC response to radiotherapy (3). However, the underlying molecular mechanisms associated with NPC radioresistance are poorly defined.

MicroRNAs (miRNAs) are small, noncoding (NC) RNAs that bind to the 3 '-untranslated regions (3'UTRs) of other messenger RNAs (mRNAs) and regulate mRNA degradation or repress their translation (4). Several miRNAs have been identified as regulators of NPC radioresistance (5-7), so we screened the miRNA database, Target Scan, and identified that miR-383-5p was a predicted upstream modulator of NPC radioresistance that targets the 3'UTR of RBM3 mRNA. Notably, miR-383-5p, which is located in chromosome $8 \mathrm{p}$, is a prognostic indicator and inhibitor of cell proliferation in lung adenocarcinoma (8). In addition, miR-383-5p is chemosensitive in ovarian cancer (9), and may play an important role in ovarian tumors. Despite its association with various cancers, the expression pattern, clinical relevance, and functional roles of $\mathrm{miR}-383-5 \mathrm{p}$ in NPC remain unclear.

Apoptosis is a critical factor that modulates the response to radiotherapy (10). Our previous study suggested that RBM3 drives radioresistance in NPC by reducing apoptosis (3). Interestingly, ac-Jun N-terminal kinase (JNK) inhibitors can reduce radiation-induced apoptosis (11), and ERK signaling activation suppresses apoptosis in radiated cells (12). Indeed, accumulating data now indicate that radiation induces cancer cell apoptosis via activation of JNK signaling and concomitant inhibition of ERK pathways; therefore, we hypothesized that miR-383-5p could attenuate the effects of RBM3 and reduce radiation-induced apoptosis through regulation of ERK and JNK signaling.

For the present study, we initially analyzed the endogenous expression of miR-383-5p between RS (Radiosensitivity) and RR (Radioresistance) NPC patienttissue specimens and cell lines. We then explored the effects of miR-383-5p on NPC cell proliferation and radioresistance, and the relationship between miR-383-5p and RBM3. Finally, we investigated the role of miR-383-5p on RBM3-mediated JNK and ERK signaling pathways in the context of NPC cell proliferation and radioresistance.

We present the following article in accordance with the ARRIVE reporting checklist (available at http://dx.doi. org/10.21037/atm-20-6881).

\section{Methods}

\section{Cell and tissue specimens}

The human NPC cell lines, CNE1 and CNE2 were obtained from the Shanghai Institutes for Biological Sciences (Chinese Academy of Sciences, Shanghai, China). CNE1 and CNE2 cells were radiated with X-ray doses of 2 Gy on non-consecutive days for 40 days, which resulted in a total of 20 radiation treatments. Viable cells after the 20 treatments were considered as radioresistant (CNE1/IR; CNE2/IR) and were cultured as previously described (13). Specimens were also obtained from 20 patients and they were classified as radiosensitive (10 cases) with no residual tumor or radioresistance (10 cases) with viable tumors following radiation therapy (70-75 Gy) (14). All procedures performed in this study involving human participants were in accordance with the Declaration of Helsinki (as revised in 2013). Informed consent was taken from all the patients. This study was approved by the Ethics Committee of Xijing Hospital (Approval ID No.YS20191010-C-1).

\section{Real-time quantitative PCR (qRT-PCR)}

Total RNA from tumor tissues and NPC cells was extracted using the RNA simple Total RNA Kit (TIANGEN, Beijing, China). The miRNAs from tumor tissues and NPC cells were extracted using the miRcute miRNA Isolation Kit (TIANGEN, Beijing, China). Reverse transcription of RBM3 and miR-383-5p was performed using the FastKing gDNA Dispelling RT SuperMix and the miRcute Plus miRNA First-Strand cDNA Kit (TIANGEN, Beijing, China), according to the manufacturer's instructions. All qPCR reactions were performed using either the SuperReal PreMix Plus (SYBR Green) or the miRcute Plus miRNA qPCR Kit (SYBR Green; TIANGEN). The primer sequence for RBM3 has been described previously (3): RBM35'-TGGGAGGGCTCAACTTTAAC3'/5'-ATGCTCTGGGTTGGTGAAG-3'. Small nuclear U6 RNA was used as an internal control for the PCR 
reactions. Primers were purchased from TIANGEN and the miRNA-383-5p primer had the following sequence: AGAUCAGAAGGUGAUUGUGGCU. The relative miR383-5p and RBM3 mRNA expression levels in paired tissues were analyzed using the comparative $2^{-\Delta \Delta \mathrm{CT}}$ method (15).

\section{Cell proliferation analysis}

NPC cells $\left(1 \times 10^{3}\right)$ were seeded in $96-$ well plates. Cell viability on days $1,3,5$ and 7 was measured with the Cell Counting Kit-8 (CCK-8, Beyotime) assay according to the manufacturer's instructions. Absorbance at $450 \mathrm{~nm}$ was measured, and each experiment was performed in triplicate.

\section{Clonogenic survival assay}

NPC cells were transfected with a miR-383-5p mimic, an inhibitor, or the respective control. At $48 \mathrm{~h}$ posttransfection, the cells were seeded in triplicate in 6-well plates and incubated overnight at $37{ }^{\circ} \mathrm{C}$ in a humidified chamber that contained $5 \% \mathrm{CO}_{2}$. The following day, the cells were irradiated with $0,2,4,6,8$, or 10 Gy. The cells were then incubated for 2 weeks to allow for macroscopic colony formation. Next, the cells were fixed for $10 \mathrm{~min}$ with $95 \%$ methanol and stained with $0.5 \%$ crystal violet for $20 \mathrm{~min}$. The number of colonies that contained $>50$ viable cells in each treatment group was counted. Survival was then calculated using the following formula: [number of colonies counted/ (number of cells seeded $\times$ plating efficiency/100)]. The clonogenic survival assay was performed in triplicate and the results are reported as the mean \pm standard deviation. The survival fractions (SF) after the indicated IR doses were used to determine radiosensitivity. GraphPad Prism 5.0 Software (GraphPad Software Inc., La Jolla, CA, USA) was used to calculate and fit the dose survival curve using a linear-quadratic model.

\section{Bioinformatics prediction}

The target genes of miR-383-5p were predicted with the assistance of the computer-aided algorithm, Target Scan Human Release 7.0 (http://www.targetscan. org/).

\section{Dual-luciferase reporter assay}

To examine whether miR-383-5p directly targets RBM3, we constructed a wild-type RBM3 3'UTR reporter plasmid (RBM3-3'UTR-wt) and a mutated-type RBM3 3'UTR reporter plasmid (RBM3-3'UTR-Mu). Both plasmids contained a pGL3-promoter. CNE1-IR cells were seeded and cultured in 24-well plates (Corning, NY, USA) for $24 \mathrm{~h}$. The CNE1-IR cells were then transfected with either the pGL3- RBM3-3'UTR-wt or the pGL3-RBM3-3'UTR-Mu plasmid, or cotransfected with miR-383-5p noncoding (NC) or mimic sequences and a plasmid. The luciferase assay was performed $48 \mathrm{~h}$ after transfection with the Dual-Luciferase Reporter Assay System (Promega, Madison, WI, USA). The relative luciferase activity was expressed as the ratio of firefly luciferase activity to Renilla luciferase activity.

\section{Flow cytometric analysis of cell apoptosis}

CNE1/IR cells $\left(5 \times 10^{4}\right.$ cells/well) were seeded in 6-well plates and cultured overnight. At $12 \mathrm{~h}$ after transfection with miR-383-5p mimic or NC sequences, the cells were radiated (6 Gy). Subsequently, $1-5 \times 10^{5}$ cells were collected by centrifugation, followed by incubation with annexin V/ (PI). The cells were then washed twice with phosphatebuffered saline and resuspended in $500 \mu \mathrm{L}$ of binding buffer. After 5-10 min of incubation with $5 \mu \mathrm{L}$ of annexin $\mathrm{V}$ and $10 \mu \mathrm{L}$ of PI, the cells were analyzed with flow cytometry. Early apoptotic cells were stained with annexin $\mathrm{V}$ alone, and necrotic and late apoptotic cells were stained with both annexin $\mathrm{V}$ and PI.

\section{Western blotting}

The treated NPC cells were lysed and the total protein content in the samples was measured with the bicinchoninic acid (BCA) Protein Assay kit (Thermo Scientific, MA, USA). A 10\% Tris-glycine Sodium dodecyl sulfate (SDS) polyacrylamide gel was used for electrophoresis followed by protein transfer to a Polyvinylidene Fluoride (PVDF) membrane. The membrane was blocked with $1 \%$ bovine serum albumin in Tris-buffered saline with $0.1 \%$ Tween-20 (TBST) for $1 \mathrm{~h}$ and subsequently incubated overnight at $4{ }^{\circ} \mathrm{C}$ with anti-RBM3 (1:1,000), anti-JNK1/2 (1:500), antip-JNK1/2 (1:1,000), anti-ERK1/2 (1:1,000), anti-p-ERK1/2 (1:1,000), anti-Bcl-2 (1:1,000), anti-Caspase3 $(1: 1,000)$, and anti- $\beta$-actin (1:500) antibodies at $4{ }^{\circ} \mathrm{C}$ overnight. All primary antibodies were purchased from Cell Signaling Technology (CST). After three washes with TBST, the membrane was incubated for $1 \mathrm{~h}$ with a 1:5,000 dilution of a horseradish peroxidase-conjugated IgG antibody in TBST. After three washes with TBST, the protein bands were detected using a Molecular Imager ChemiDoc XRS System 

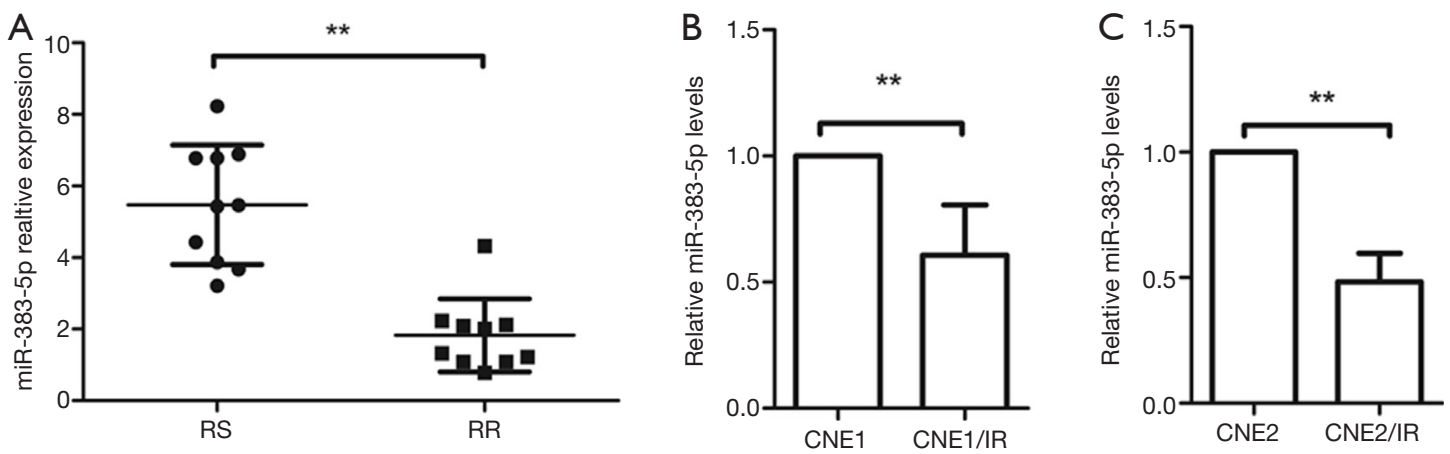

Figure 1 Evaluation of miR-383-5p expression in radioresistant samples and cell lines of nasopharyngeal carcinoma (NPC). (A) qRT-PCR analysis of miR-383-5p was performed in NPC samples ( $\mathrm{n}=10$ pairs of RR and RS tissues). The expression was normalized to human U6. The expression of miR-383-5p was lower in RR NPC samples compared with RS. (B,C) Relative expression of miR-383-5p in two pairs of RR and RS NPC cell lines (CNE1 and CNE1/IR; CNE2 and CNE2/IR) via using qRT-PCR. The expression was normalized to human U6. The expression of miR-383-5p was lower in RR NPC cell lines (CNE1/IR; CNE2/IR) compared with parental NPC cell lines (CNE1; CNE2). Data are presented at the mean \pm standard deviation ${ }^{* *} \mathrm{P} \leq 0.01$. RS, radiosensitivity; RR, radioresistance; NPC, nasopharyngeal carcinoma.

(Bio-Rad, Hercules, CA, USA). The band intensities were quantified using densitometry with ImageJ.

\section{In vivo xenograft tumor model}

The transplanted tumor model was developed as described in our prior work (3). Briefly, CNE1/IR cells were subcutaneously transplanted in nude mice. When the tumors reached a volume of approximately $500 \mathrm{~mm}^{3}$, they were exposed to $6 \mathrm{~Gy}$ of radiation. The mice then received weekly injections of miR-383-5p agomir or antagomir. Tumor growth was measured every 2 days and calculated according to the following formula: (length $\times$ width $\left.^{2}\right) / 2$. After 3 weeks of injections, the mice were killed by cervical dislocation, and the tumors were removed and photographed. Experiments were performed in compliance with the ethics committee of the Air Force Medical University guidelines for the care and use of animals (NO.: IACUC-20191013).

\section{Immunobistochemistry}

Unstained slides for immunohistochemical analysis were prepared from formalin-fixed and paraffin-embedded blocks of tumors tissues, and staining was performed as previously described (16). Briefly, the tumor slides were incubated with primary antibody RBM3 (1:200, Proteintech, USA), Bcl-2 (1:400, CST, USA) and Caspase3 (1:200, CST, USA) at $4{ }^{\circ} \mathrm{C}$ overnight. The reaction was stained using diaminobenzidine as the chromogen and observed under $\times 200$ magnification.

\section{Statistical analysis}

All values are expressed as the mean \pm standard deviation. A Student's $t$ test or a two-way analysis of variance (ANOVA) with a Tukey's post-hot test was used for statistical comparison, as appropriate. Statistical significance was defined as ${ }^{* *} \mathrm{P} \leq 0.01$.

\section{Results}

\section{miR-383-5p expression of NPC radiation-resistant tissue specimens and cells}

To investigate the role of miR-383-5p in NPC cell proliferation and radioresistance, we examined its expression in 10 pairs of RS and RR NPC patient-tissue specimens and in two RS and RR cell lines (CNE1 and CNE1/IR; CNE2 and CNE2/IR) by qRT-PCR. We found that miR-383$5 \mathrm{p}$ was downregulated in the RR NPC tissue specimens (Figure $1 A$ ) and cell lines (Figure $1 B, C$ ) when compared to the RS counterparts.

\section{Effects of miR-383-5p on NPC cell proliferation and radioresistance in vitro}

To further investigate the role of miR-383-5p in NPC, 
A

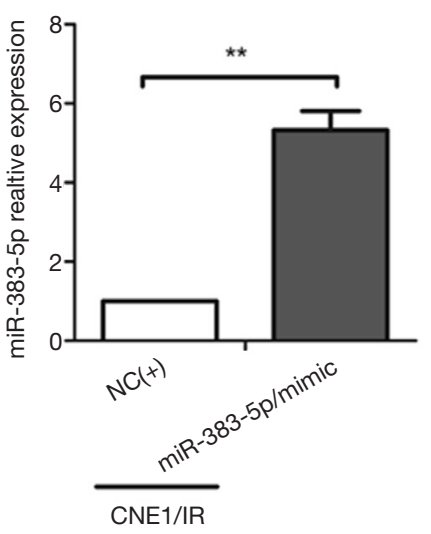

C

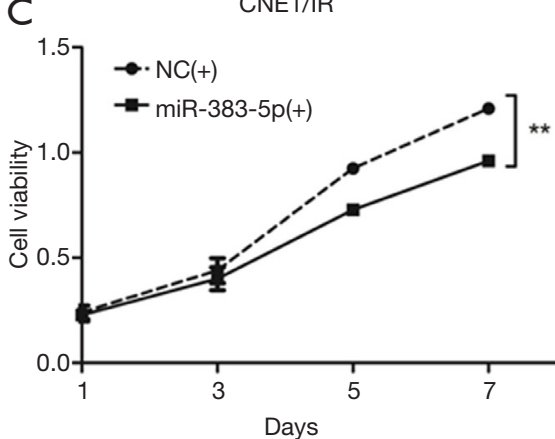

$E$

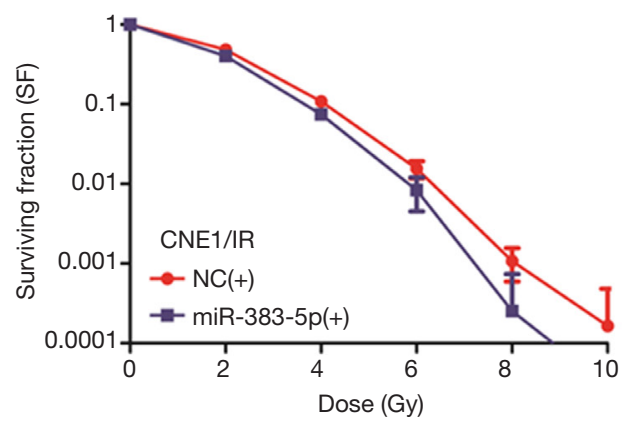

B

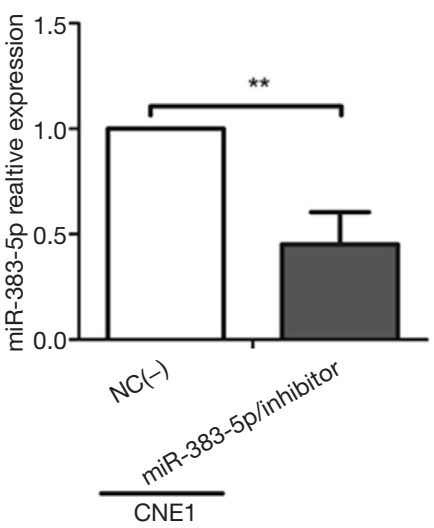

D

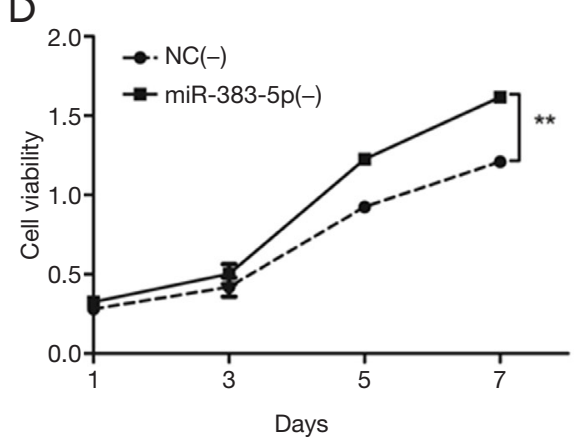

$\mathrm{F}$

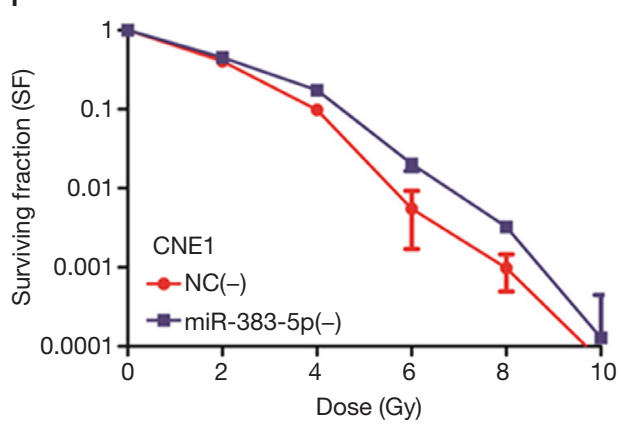

Figure 2 Effect of miR-383-5p on nasopharyngeal carcinoma (NPC) cell proliferation and radioresistance in vitro. (A,B) qRT-PCR analyses of miR-383-5p expression in CNE1/IR and CNE1 cells transfected with a miR-383-5p mimic or an inhibitor. (C,D) Cell viability was analyzed with a CCK-8 assay in CNE1/IR and CNE1 cells transfected with a miR-383-5p mimic or an inhibitor. Overexpression of miR$383-5 p$ resulted in evident inhibition of proliferation of CNE1/IR cells. The miR-383-5p inhibitor significantly promoted the growth of CNE1 cells. (E,F) miR-383-5p inhibited NPC radioresistance in vitro in the clonogenic survival assay. Overexpression of miR-383-5p using mimics clearly inhibited the surviving fraction (SF) of CNE1/IR. Knocking down miR-383-5p using chemically synthesized miR-383-5p inhibitors increased the SF of CNE1. The data are shown as the mean $\pm \mathrm{SD}$. ${ }^{* *} \mathrm{P} \leq 0.01$. NPC, nasopharyngeal carcinoma; NC, noncoding.

we transfected a miR-383-5p mimic into CNE1/IR cells and an inhibitor into CNE1 cells. The transfection was confirmed by qRT-PCR (Figure $2 A, B$ ), and the CCK-8 assay was used to analyze NPC cell proliferation. As shown in Figure 2C,D, overexpression of miR-383-5p decreased cell proliferation when compared with the control CNE1/
IR cells; In contrast, depletion of miR-383-5p increased cell proliferation in CNE1 cells. A clonogenic survival assay was used to investigate NPC radioresistance and revealed that miR-383-5p overexpression suppressed CNE1/IR cell radioresistance. In contrast, depletion of miR-383-5p increased radioresistance in CNE1 cells (Figure $2 E, F$ ). In 


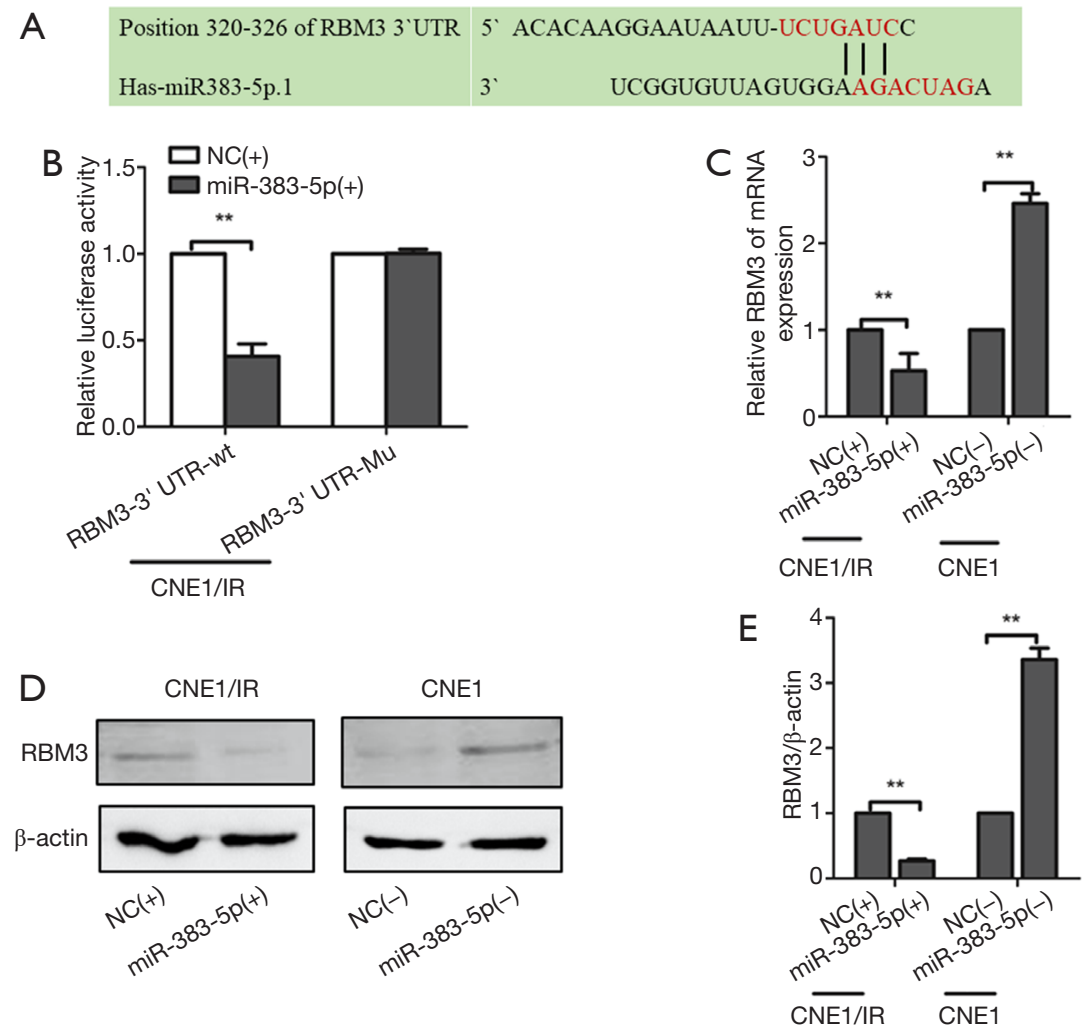

Figure 3 RBM3 is a direct target of miR-383-5p. (A) Schematic illustration of the predicted miR-383-5p-binding site in the RBM3 3'UTR. (B) Luciferase reporter assay was performed to validate that miR-383-5p targets RBM3. The reporter constructs are shown in which the 3'UTR of RBM3 with either wild-type or mutant (pGL3-RBM3-3'UTR-wt or pGL3-RBM3-3'UTR-Mu) miR-383-5p binding sites was cloned downstream of the luciferase open reading frame. CNE1/IR cells were co-transfected with the luciferase construct and miR-383-5p mimics or control miRNA (NC). Luciferase activity was normalized to Renilla luciferase activity. (C,D,E) miR-383-5p downregulated the expression of RBM3. qRT-PCR and western blotting analyses of RBM3 were performed $48 \mathrm{~h}$ after transfection of the miR-383-5p mimics and the miR-383-5p inhibitor. $\beta$-actin was used as an internal control. The data are shown as the mean $\pm \mathrm{SD}$. ${ }^{* *} \mathrm{P} \leq 0.01$. RBM3, RNA binding protein motif; miRNA, microRNA; NC, noncoding; UTR, untranslated region.

summary, our results suggested that miR-383-5p inhibited NPC cell proliferation and radioresistance.

\section{$R B M 3$ as direct target of $m i R-383-5 p$}

We screened the Target Scan miRNA database and found that miR-383-5p was predicted to target the 3'UTR of RBM3 mRNA (Figure $3 A$ ). To validate the interaction between RBM3 and miR-383-5p, we constructed luciferase reporter plasmids that contained either the wild-type or a mutated form of the 3'UTR of RBM3. The plasmids were transfected into CNE1/IR cells expressing either miR-NC or a miR-383-5p mimic, and luciferase activity was measured (Figure 3B). Overexpression of miR$383-5 \mathrm{p}$ decreased the luciferase activity of the RBM3
3'UTR in both reporter plasmids. Notably, site-directed mutagenesis abolished the interaction between miR-383$5 \mathrm{p}$ and the 3'UTR of RBM3. Subsequently, qRT-PCR and western blotting were used to analyze the expression of RBM3 mRNA and protein in miR-383-5p-overexpressing CNE1/IR and miR-383-5p-depleted CNE1 cells. The results suggested that relative expression of RBM3 was downregulated in CNE1/IR cells that overexpressed miR383-5p when compared with NC-control. In contrast, the relative expression of RBM3 was upregulated in miR-3835p-depleted CNE1 cells (Figure 3C). Correspondingly, $\mathrm{RBM} 3$ protein expression was decreased in miR-383-5poverexpressing CNE1/IR in comparison with NC-control (Figure 3D,E). Thus, these results indicated that RBM3 was a miR-383-5p target gene in NPC cells. 

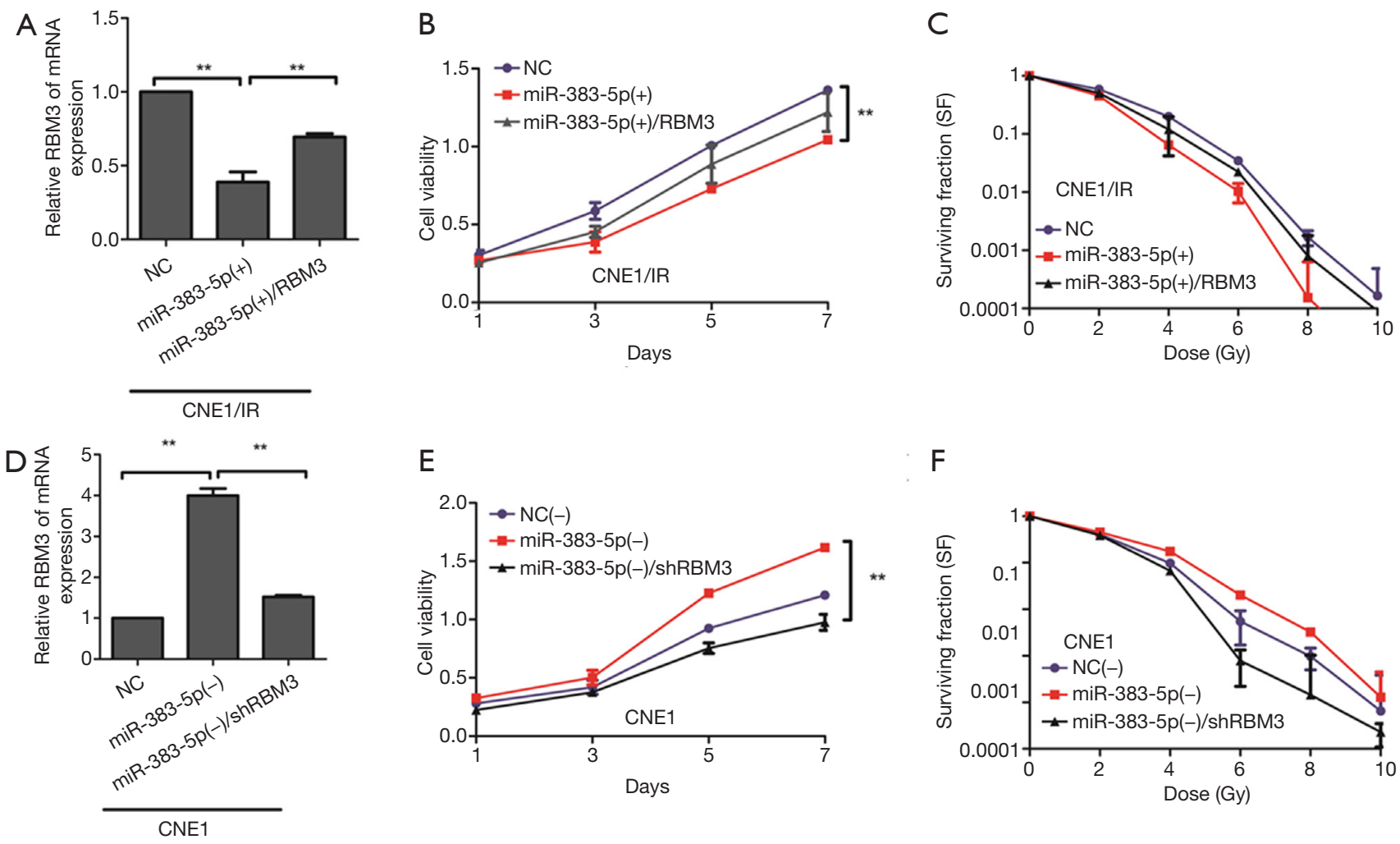

Figure 4 miR-383-5p regulates NPC cell proliferation and radioresistance through RBM3. (A) qRT-PCR analyses of RBM3 were performed into CNE1/IR cell line that were stably infected with overexpression vector pCDNA3.1-RBM3. (B,C) CCK-8 assay and clonogenic survival assay were detected cell proliferation and radioresistance in NPC cells after transfection of the miR-383-5p mimics at were stably infected with overexpression vector pCDNA3.1-RBM3, respectively. (D) The miR-383-5p inhibitor was transfected into CNE1 cell lines that were stably infected with LV16-shRBM3, and the relative expression of RBM3 was performed via qRT-PCR. (E,F) The miR-383-5p inhibitor was transfected into CNE1 cell lines that were stably infected with LV16-shRBM3. NPC cell proliferation and radio-responsiveness were measured with the CCK-8 assay and clonogenic survival assay. RBM3 reversed the inhibitory effects of miR-383-5p on radioresistance. The data are shown as the mean $\pm \mathrm{SD}$. ${ }^{* *} \mathrm{P} \leq 0.01$. RBM3, RNA binding protein motif; NPC, nasopharyngeal carcinoma; NC, noncoding.

\section{Location of inbibition of NPC cell proliferation and radioresistance through RBM3 regulation}

We performed a series of rescue experiments to verify that miR-383-5p regulated RBM3. A miR-383-5p mimic was transfected into CNE1/IR cell lines that were stably infected with overexpression vector pCDNA3.1-RBM3, the expression of RBM3 was confirmed by qRT-PCR (Figure $4 A$ ). Analysis of a proliferation assay and a clonogenic survival assay demonstrated that overexpression of RBM3 reversed the inhibitory effects of miR-383-5p on cell proliferation and radioresistance (Figure 4B,C). In contrast, a miR-383-5p inhibitor was transfected into CNE1 cell lines that were stably infected with LV16-shRBM3. The expression of RBM3 was confirmed by qRT-PCR (Figure $4 D$ ). Moreover, proliferation and radioresistance were significantly reduced by RBM3 shRNA in miR-3835p downregulated CNE1 cells (Figure 4E,F). Together, these results showed that miR-383-5p inhibited NPC cell proliferation and radioresistance by regulating RBM3.

\section{Effect of miR-383-5p on radiation-induced apoptosis}

To analyze the effect of miR-383-5p expression on NPC cell apoptosis caused by radiation, we performed a flow cytometric assay and found that the overexpression of miR-383-5p promoted radiation-induced apoptosis in CNE1-IR cells (Figure 5A), but miR-383-5p silencing had the opposite effect on CNE1 cells (Figure 5B). To further investigate the role of miR-383-5p on radiation-induced apoptosis, western blotting was used to detect two apoptosis-related proteins: $\mathrm{Bcl}-2$, an 


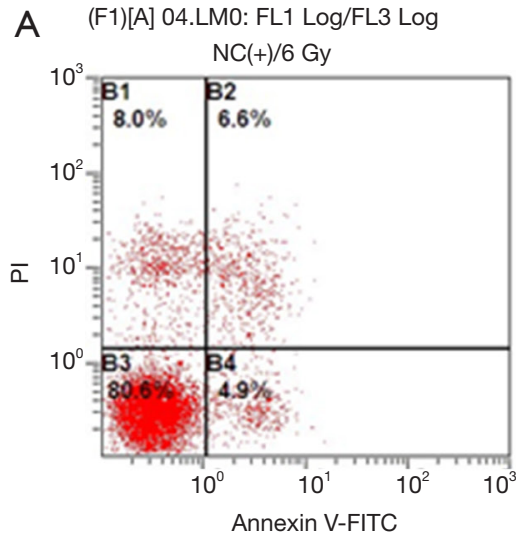

B (F1)[A] 2.LM0: FL1 Log/FL3 Log

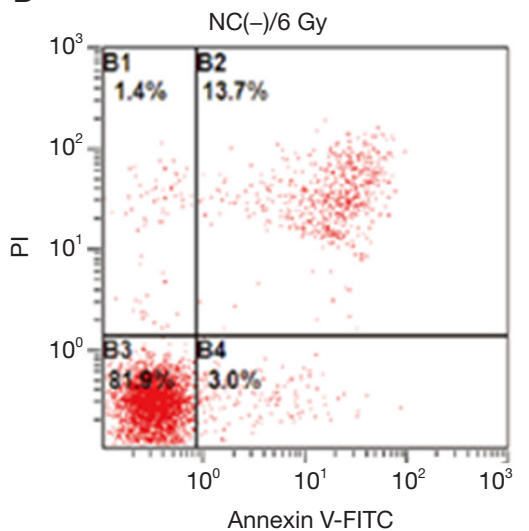

C
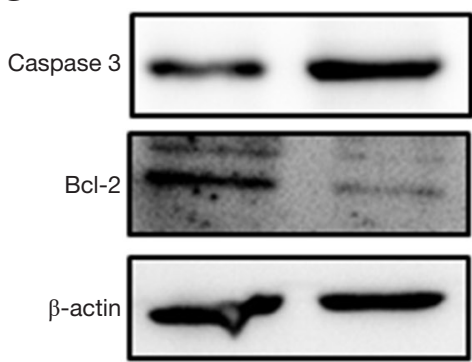

$n C(x)$

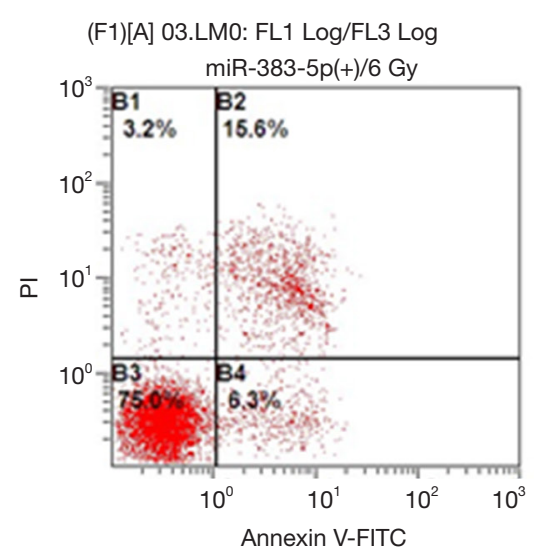

(F1)[A] 1.LM0: FL1 Log/FL3 Log

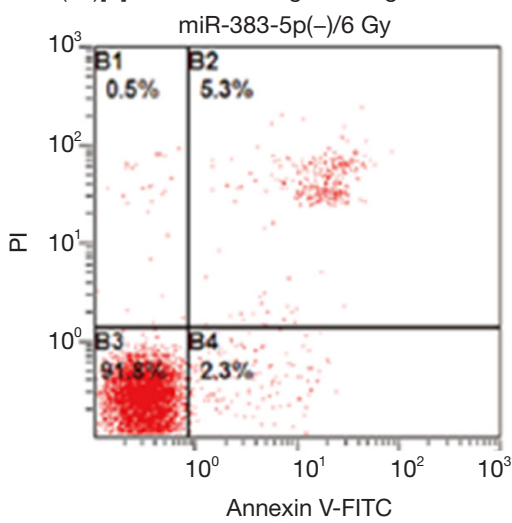

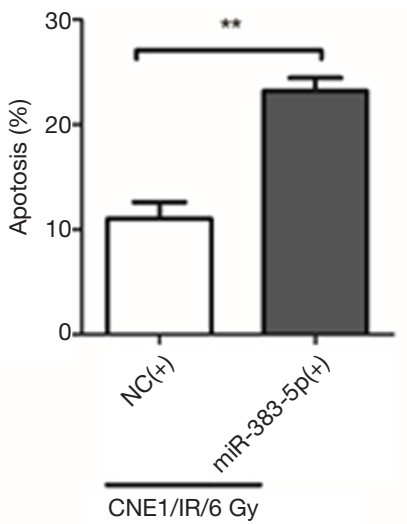

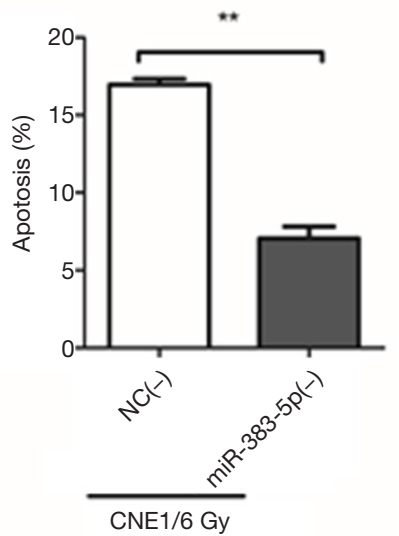

CNE1/6 Gy
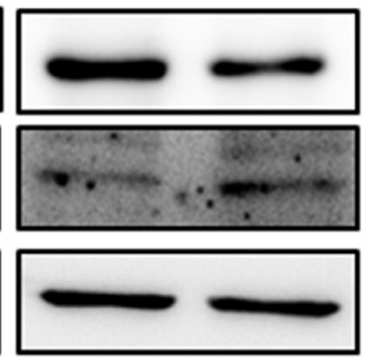

$n \operatorname{mit}^{-38^{3-5 p^{-\lambda}}}$

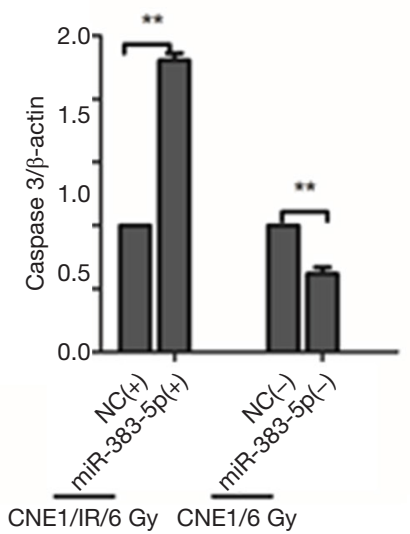

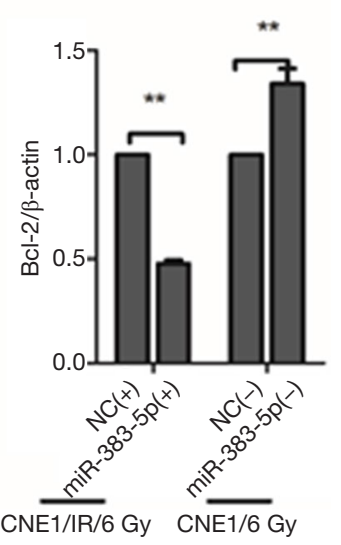

Figure 5 Radiation-induced apoptosis is enhanced by miR-383-5p. (A,B) Apoptosis was analyzed with flow cytometry in nasopharyngeal carcinoma (NPC) cells that were transfected with miR-383-5p or an inhibitor and treated with 6 Gy of radiation. miR-383-5p increased radiation-induced apoptosis. (C) Western blotting analyses of Bcl-2 and Caspase3 were performed $48 \mathrm{~h}$ after transfection of the miR-383$5 \mathrm{p}$ mimics or inhibitor (X-ray $6 \mathrm{~Gy}$ ). $\beta$-actin was used as an internal control. Image $\mathrm{J}$ software was used for densiometric quantification. The data are shown as the mean $\pm \mathrm{SD} .{ }^{* *} \mathrm{P} \leq 0.01$. FITC, fluorescein isothiocyanate; NC, noncoding.

anti-apoptotic protein, and Caspase3, a pro-apoptotic protein. The data revealed that $\mathrm{Bcl}-2$ expression was downregulated in CNE1/IR-radiated cells that were transfected with the miR- 383-5p mimic. In contrast, Bcl-2 expression was upregulated in CNE1 cells that were transfected with the miR-383-5p inhibitor. We also observed increased Caspase 3 expression in 

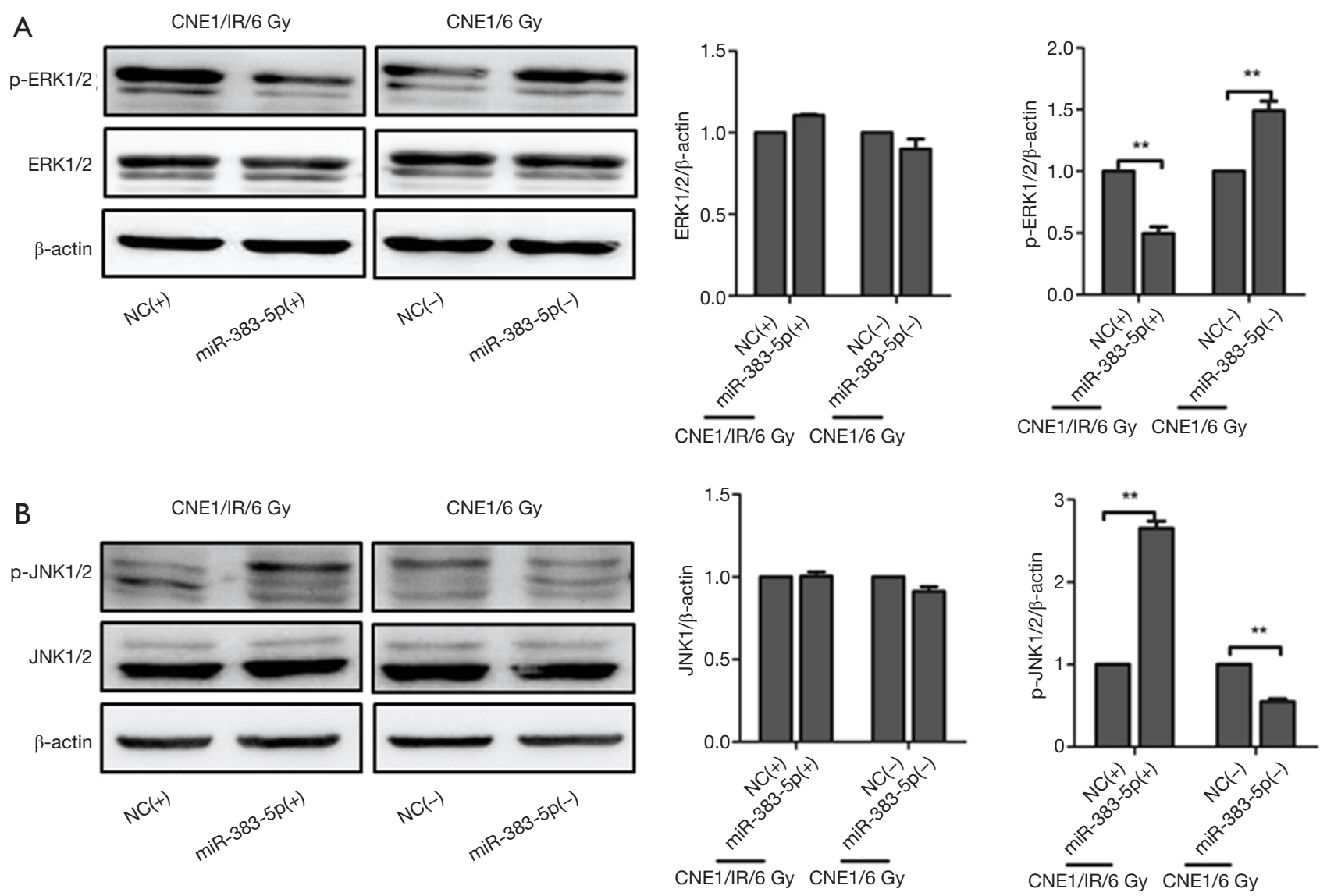

Figure 6 JNK activation and ERK inhibition occur downstream of miR-383-5p. (A) Western blot analyses of ERK1/2 and p-ERK1/2 was performed $48 \mathrm{~h}$ after transfection of the miR-383-5p mimics or inhibitor and treated with 6 Gy of radiation. (B) Western blotting analyses of JNK1/2 and p-JNK1/2 was performed $48 \mathrm{~h}$ after transfection of the miR-383-5p mimics or inhibitor and treated with 6 Gy of radiation. The levels of JNK1/2and p-JNK1/2, ERK1/2 and p-ERK1/2 was quantified by densitometry with Image J software. The data are shown as the mean $\pm \mathrm{SD} .{ }^{* *} \mathrm{P} \leq 0.01$. NC, noncoding.

CNE1/IR-irradiated cells that were transfected with the miR383-5p mimic and decreased Caspase3 expression with the miR-383-5p inhibitor in CNE1/IR-radiated cells (Figure 5C). These results suggested that miR-383-5p increased radiationinduced apoptosis.

\section{miR-383-5p inbibition of NPC cell proliferation and radioresistance via alterations in $7 \mathrm{NK}$ and ERK signaling patbways}

Accumulating evidence suggests that radiation-induced cancer cell apoptosis is regulated by the JNK and ERK signaling pathways; therefore, we hypothesized that these signaling pathways could be the downstream targets of miR-383-5p. Upregulation of miR-383-5p in CNE1/IR cells led to enhanced JNK activation and simultaneously inhibited the ERK signaling pathway. In agreement with this data, overexpression of miR-383-5p in CNE1/IR cells contributed to a significant increase in the radiation-induced activation of JNK (171.3\% increase) and inhibited the radiation-induced activation of ERK (55.4\% reduction). By comparison, the CNE1 cells with downregulated miR-383$5 \mathrm{p}$ had decreased radiation-induced JNK activation (42.6\% reduction) and increased radiation-induced activation of ERK (43.5\% increase) (Figure 6A,B). In summary, the data suggested that miR-383-5p inhibited NPC cell proliferation and radioresistance by altering ERK and JNK signaling.

\section{Effect of miR-383-5p on in vivo NPC cell proliferation and radioresistance}

To investigate the in vivo effects of miR-383-5p on NPC 
radioresistance, we performed subcutaneous transplantation of CNE1/IR cells in nude mice $(n=6)$. When the tumors were approximately $500 \mathrm{~mm}^{3}$, they were injected with a NC-control, a miR-383-5p agomir, or a miR-383$5 \mathrm{p}$ antagomir and exposed to $6 \mathrm{~Gy}$ of ionizing radiation once weekly. After 3 weeks, the tumors were excised and photographed. As shown in Figure 7A, the miR-383-5p agomir-injected tumors had significantly less radioresistance than control injected tumors. The radioresistant tumors exhibited attenuated growth. Conversely, the tumors that were injected with the miR-383-5p antagomir displayed increased radioresistance when compared with the $\mathrm{NC}$ group (Figure 7B). Subsequent immunohistochemical staining results suggested that the expression level of RBM3 in the xenograft tumors with injecting the miR-383-5p agomir was significantly decreased compared with control injected tumors. Conversely, the expression level of RBM3 in the xenograft tumors with injecting the miR-383-5p antagomir was obvious increased compared to the control injected tumors. (Figure 7C). Immunohistochemical staining for Caspase3 was detected to be higher with injecting the miR-383-5p agomir compared with control injected tumors (Figure 7D), whereas immunohistochemical staining for Bcl-2 had the opposite result (Figure 7E). Collectively, our results suggested that miR-383-5p inhibited NPC cell proliferation and radioresistance.

\section{Discussion}

Radioresistance is the primary obstacle in the clinical management of NPC (17). Accordingly, radioresistance biomarkers and the underlying molecular mechanisms that contribute to NPC radioresistance have been analyzed (18). For example, our previous study identified that RBM3 was associated with radioresistance in NPC (3), but the complete mechanism by which RBM3 contributes to NPC radioresistance is unknown.

In the current study, we focused on miR-383-5p, which is one of the RBM3 upstream regulatory molecules found in radioresistant NPC cells. Because miRNAs are small, NC regulatory RNAs, they play crucial roles in almost all tumor processes and act as both oncogenes and tumor suppressors in malignant tumors (19). Mounting evidence suggests that several miRNAs are involved in NPC tumor cell proliferation and radioresistance (20-26). Recent studies report that dysregulation of miR-383 is associated with various cancers, including hepatocellular carcinoma (27), ovarian cancer (9), and lung cancer (28). However, the precise role of miR-383 in NPC is not fully understood. In our study, we found that miR-383-5p was downregulated in RR clinical samples and cells. In addition, miR-383-5p inhibited NPC cell proliferation and radioresistance in vitro and in vivo. These results suggested that miR-383-5p has a crucial role in RBM3-mediated NPC cell proliferation and radioresistance.

Because miRNAs can inhibit the translation of mRNA, the identification of miR-383-5p target genes is a key step in understanding the mechanisms of NPC cell proliferation and radioresistance. In the present study, we used bioinformatics and a luciferase reporter assay to identify that RBM3 is a direct target of miR-383-5p in NPC cells. Furthermore, our data indicated that miR-383$5 \mathrm{p}$ regulated NPC cell proliferation and radioresistance by targeting RBM3, which is an RNA binding-motif protein that contributes to NPC radioresistance by reducing apoptosis (3). Importantly, apoptosis is essential to the development and progression of NPC and also confers tumor resistance to radiotherapy (29). Bucci and colleagues found that fractionated ionizing radiation induced apoptosis through Caspase 3 activation (30). Based on these prior findings, we investigated whether miR-383-5p enhanced radiation-induced apoptosis in NPC cells. Our data demonstrated that a miR-383-5p mimic enhanced radiation-induced apoptosis in NPC, whereas a miR-383-5p inhibitor had the opposite effect. Taken together, our results revealed that miR-383-5p inhibited NPC cell proliferation and radioresistance through RBM3 to promote radiationinduced apoptosis.

Both JNK and ERK are known to have an important role in cancer development (31). Interestingly, unlike most cancers, NPC exhibits induced JNK regulation via a latent membrane protein 1(LMP1)-dependentroute (32). Prolonged JNK activation is a hallmark of both NPC and oral squamous cell carcinoma $(33,34)$. Indeed, constitutive activation of JNK in NPC promotes cancer development and JNK inhibitors have been investigated as a method of reducing radiation-induced apoptosis $(30,35)$. Despite these prior studies, the JNK expression pattern and its role in NPC radioresistance is unclear. In the current study, we detected the expression of JNK1/2 and p-JNK1/2 in NPC cells that were transfected with a miR-383-5p mimic or inhibitor and exposed to $6 \mathrm{~Gy}$ of $\mathrm{X}$-ray radiation. Interestingly, we found that overexpression of miR-383-5p in CNE1/IR cells significantly enhanced radiation-induced JNK activation, whereas miR-383-5p downregulation in CNE1 cells inhibited radiation-induced JNK activation. 
A
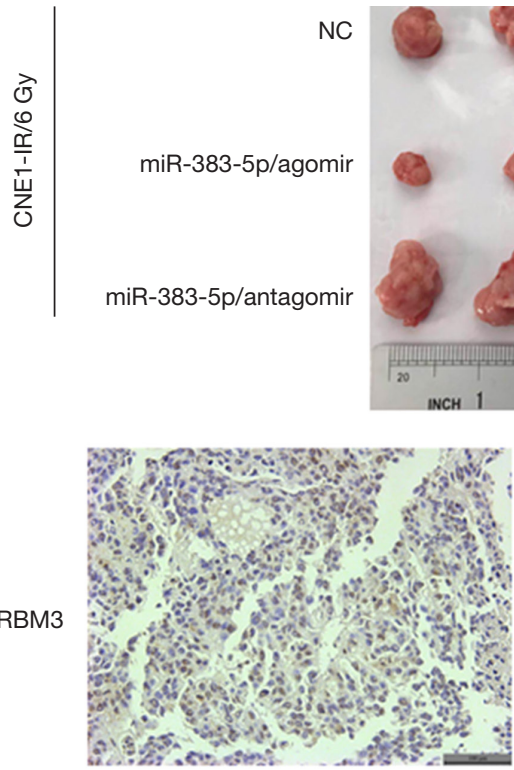

NC

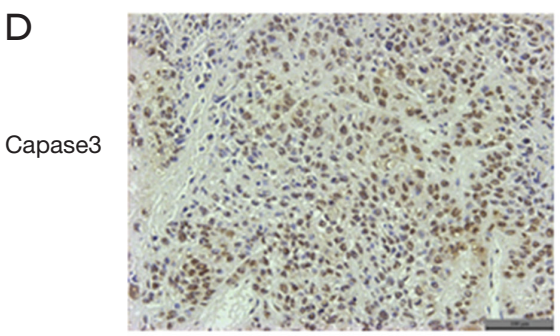

NC

$E$

$\mathrm{Bcl}-2$

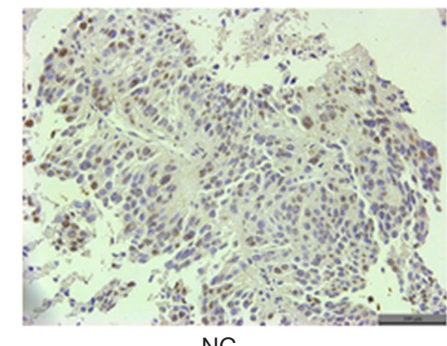

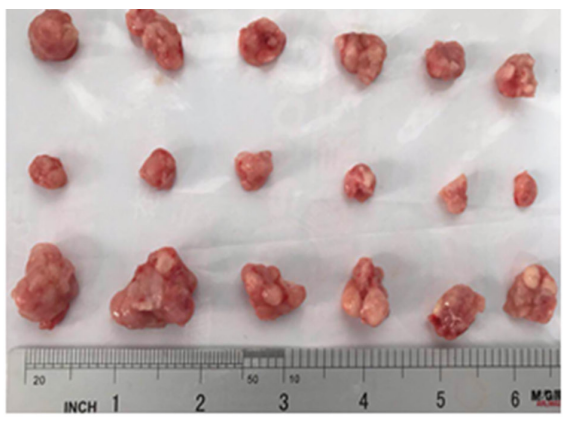

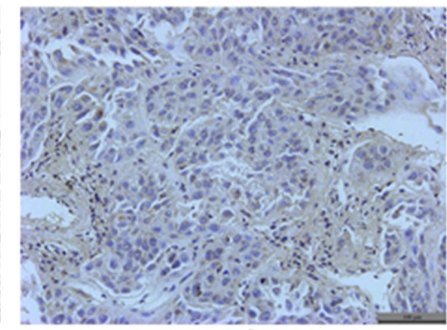

Agom1r

CNE1-IR/6 Gy

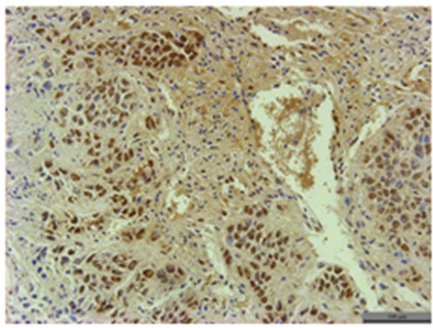

Agom1r

CNE1-IR/6 Gy

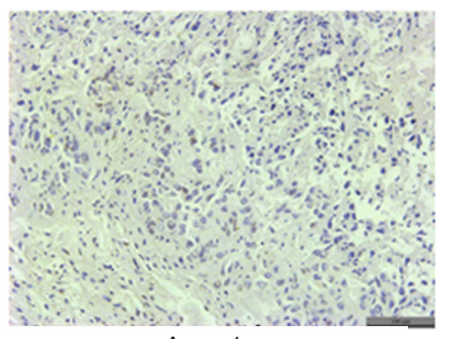

Agom1r
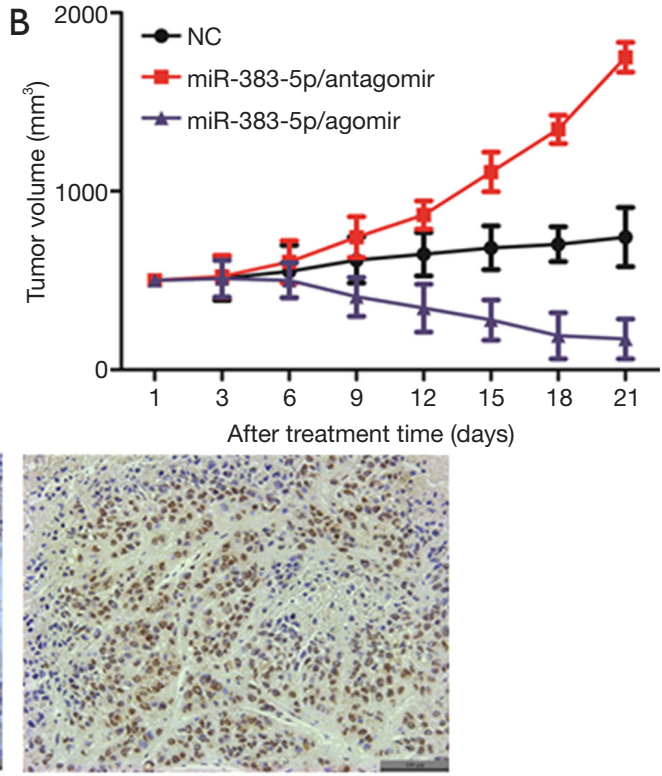

Antagomir

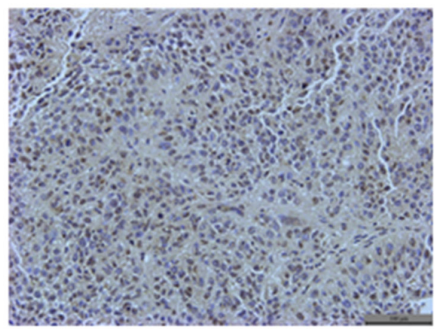

Antagomir

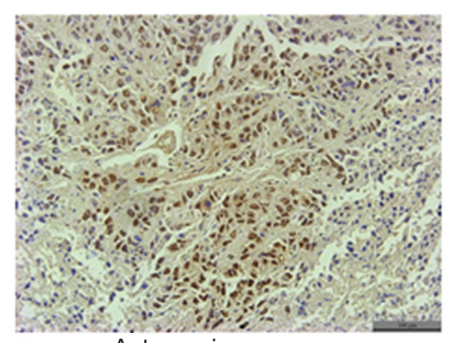

Antagomir

CNE1-IR/6 Gy

Figure 7 In vivo inhibition of NPC cell radioresistance by miR-383-5p. (A) Tumor growth of miR-383-5p agomir- or antagomirtreated CNE1/IR xenografts treated with 6 Gy of radiation in nude mice ( $\mathrm{n}=6$ each). The tumors excised from the mice are shown. (B) Tumor growth curves were calculated and indicated that the tumor growth rate was higher in cells treated with the miR-383-5p antagomir when compared with the negative control. Tumors treated with the miR-383-5p agomir exhibited decreased growth. (C) RBM3 expression in tumors was analyzed by immunohistochemistry (magnification: $\times 200$ ). (D) Caspase 3 expression in tumors was analyzed by immunohistochemistry (magnification: $\times 200$ ). (E) Bcl-2 expression in tumors was analyzed by immunohistochemistry (magnification: $\times 200$ ). The data are shown as the mean $\pm \mathrm{SD}$. ${ }^{* *} \mathrm{P} \leq 0.01$. RBM3, RNA binding protein motif; NPC, nasopharyngeal carcinoma; NC, noncoding. 
The ERK pathway also regulates NPC progression, and individual pathways components have been explored as potential biomarkers for NPC prognosis(36). Important to our study, the activation of ERK signaling suppressed apoptosis in irradiated cells. We analyzed ERK1/2 and p-ERK1/2 in NPC cells that were transfected with amiR$383-5 \mathrm{p}$ mimic or a corresponding inhibitor and exposed to 6 Gy of X-ray radiation. We observed that miR-383-5p overexpression in CNE1/IR cells inhibited ERK activation caused by radiation. In summary, the data suggested that miR-383-5p activated JNK and inhibited ERK signaling pathways in NPC RR cells. From a therapeutic perspective, targeting miR-383-5p and RBM3-mediated radioresistance may be a promising strategy for NPC.

\section{Conclusions}

In conclusion, our data demonstrated that miR-383-5p is expressed at low levels in RR NPC cells and clinical samples. In addition, miR-383-5p inhibited NPC cell proliferation and radioresistance in vitro and in vivo through targeting RBM3. Enhanced miR-383-5p expression increased radiation-induced apoptosis. We also observed that miR-383-5p could alter signaling, and that it activated and inactivated the JNK and ERK signaling pathways, respectively. Our study demonstrated that miR-383$5 \mathrm{p}$ is a critical regulator of NPC cell proliferation and radioresistance. These data suggest that targeting the miR383-5p/RBM3/JNK/ERK signaling axis is a promising approach to enhancing NPC sensitivity to radiotherapy. However, the specific mechanism by which miR-383-5p inhibits NPC cell proliferation and radioresistance through JNK and ERK is still unclear and necessitates additional studies.

\section{Acknowledgments}

Funding: This work was supported by the National Natural Science Foundation of China (grant no. 81872699, grant nos. 81372421 and 81802977$)$.

\section{Footnotes}

Reporting Checklist: The authors have completed the ARRIVE reporting checklist. Available at http://dx.doi. org/10.21037/atm-20-6881

Data Sharing Statement: Available at http://dx.doi. org/10.21037/atm-20-6881

Conflicts of Interest: All authors have completed the ICMJE uniform disclosure form (available at http://dx.doi. org/10.21037/atm-20-6881). The authors have no conflicts of interest to declare.

Ethical Statement: The authors are accountable for all aspects of the work in ensuring that questions related to the accuracy or integrity of any part of the work are appropriately investigated and resolved. All procedures performed in this study involving human participants were in accordance with the Declaration of Helsinki (as revised in 2013). Informed consent was taken from all the patients. This study was approved by the Ethics Committee of XiJing Hospital (Approval ID No.YS20191010-C-1). Experiments were performed in compliance with the ethics committee of the Air Force Medical University guidelines for the care and use of animals (NO.: IACUC-20191013).

Open Access Statement: This is an Open Access article distributed in accordance with the Creative Commons Attribution-NonCommercial-NoDerivs 4.0 International License (CC BY-NC-ND 4.0), which permits the noncommercial replication and distribution of the article with the strict proviso that no changes or edits are made and the original work is properly cited (including links to both the formal publication through the relevant DOI and the license). See: https://creativecommons.org/licenses/by-nc-nd/4.0/.

\section{References}

1. Lo KW, To KF, Huang DP. Focus on nasopharyngeal carcinoma. Cancer Cell 2004;5:423-8.

2. Luftig M. Heavy LIFting: tumor promotion and radioresistance in NPC. J Clin Invest 2013;123:4999-5001.

3. Ma R, Zhao LN, Yang $\mathrm{H}$, et al. RNA binding motif protein 3 (RBM3) drives radioresistance in nasopharyngeal carcinoma by reducing apoptosis via the PI3K/AKT/Bcl-2 signaling pathway. Am J Transl Res 2018;10:4130-40.

4. Bartel DP. MicroRNAs: genomics, biogenesis, mechanism, and function. Cell 2004;116:281-97.

5. Li LN, Xiao T, Yi HM, et al. MiR-125b Increases Nasopharyngeal Carcinoma Radioresistance by Targeting A20/NF-kappaB Signaling Pathway. Mol Cancer Ther 2017;16:2094-106.

6. Feng X, Lv W, Wang S, et al. miR495 enhances the efficacy of radiotherapy by targeting GRP78 to regulate 
EMT in nasopharyngeal carcinoma cells. Oncol Rep 2018;40:1223-32.

7. Spence T, Bruce J, Yip KW, et al. MicroRNAs in nasopharyngeal carcinoma. Chin Clin Oncol 2016;5:17.

8. Zhao S, Gao X, Zang S, et al. MicroRNA-383-5p acts as a prognostic marker and inhibitor of cell proliferation in lung adenocarcinoma by cancerous inhibitor of protein phosphatase 2A. Oncol Lett 2017;14:3573-9.

9. Jiang J, Xie C, Liu Y, et al. Up-regulation of miR-383-5p suppresses proliferation and enhances chemosensitivity in ovarian cancer cells by targeting TRIM27. Biomed Pharmacother 2019;109:595-601.

10. Fulda S. Targeting IAP proteins in combination with radiotherapy. Radiat Oncol 2015;10:105.

11. Wu LH, Li P, Zhao QL, et al. Arbutin, an intracellular hydroxyl radical scavenger, protects radiation-induced apoptosis in human lymphoma U937 cells. Apoptosis 2014;19:1654-63.

12. Hein AL, Ouellette MM, Yan Y. Radiation-induced signaling pathways that promote cancer cell survival (review). Int J Oncol 2014;45:1813-9.

13. Ma R, Zhao LN, Yang H, et al. RNA binding motif protein 3 (RBM3) drives radioresistance in nasopharyngeal carcinoma by reducing apoptosis via the PI3K/AKT/Bcl-2 signaling pathway. Am J Transl Res 2018;10:4130-40.

14. Miyata H, Doki Y, Shiozaki H, et al. CDC25B and p53 are independently implicated in radiation sensitivity for human esophageal cancers. Clin Cancer Res 2000;6:4859-65.

15. Watson JD GA, Baker TA. Molecular biology of gene. New York: Cold Spring Harbor Laboratory Press, 2013.

16. Wu CC, Li H, Xiao Y, et al. Over-expression of IQGAP1 indicates poor prognosis in head and neck squamous cell carcinoma. J Mol Histol 2018;49:389-98.

17. Kristensen CA, Kjaer-Kristoffersen F, Sapru W, et al. Nasopharyngeal carcinoma. Treatment planning with IMRT and 3D conformal radiotherapy. Acta Oncol 2007;46:214-20.

18. Chen W, Hu GH. Biomarkers for enhancing the radiosensitivity of nasopharyngeal carcinoma. Cancer Biol Med 2015;12:23-32.

19. Frixa T, Donzelli S, Blandino G. Oncogenic MicroRNAs: Key Players in Malignant Transformation. Cancers (Basel) 2015;7:2466-85.

20. Huang Y, Tan D, Xiao J, et al. miR-150 contributes to the radioresistance in nasopharyngeal carcinoma cells by targeting glycogen synthase kinase-3beta. J Cancer Res Ther 2018;14:111-8.

21. Kang M, Xiao J, Wang J, et al. MiR-24 enhances radiosensitivity in nasopharyngeal carcinoma by targeting SP1. Cancer Med 2016;5:1163-73.

22. Qu C, Liang Z, Huang J, et al. MiR-205 determines the radioresistance of human nasopharyngeal carcinoma by directly targeting PTEN. Cell Cycle 2012;11:785-96.

23. Sun Q, Liu T, Zhang T, et al. MiR-101 sensitizes human nasopharyngeal carcinoma cells to radiation by targeting stathmin 1. Mol Med Rep 2015;11:3330-6.

24. Wang S, Pan Y, Zhang R, et al. Hsa-miR-24-3p increases nasopharyngeal carcinoma radiosensitivity by targeting both the 3'UTR and 5'UTR of Jab1/CSN5. Oncogene 2016;35:6096-108.

25. Wu W, Chen X, Yu S, et al. microRNA-222 promotes tumor growth and confers radioresistance in nasopharyngeal carcinoma by targeting PTEN. Mol Med Rep 2018;17:1305-10.

26. Zhu H, Zhu X, Cheng G, et al. Downregulation of microRNA-2 1 enhances radiosensitivity in nasopharyngeal carcinoma. Exp Ther Med 2015;9:2185-9.

27. Chen L, Guan H, Gu C, et al. miR-383 inhibits hepatocellular carcinoma cell proliferation via targeting APRIL. Tumour Biol 2016;37:2497-507.

28. Ma H, Liu B, Wang S, et al. MicroRNA-383 is a tumor suppressor in human lung cancer by targeting endothelial PAS domain-containing protein 1. Cell Biochem Funct 2016;34:613-9.

29. Xia Y, Wei K, Yang F-M, et al. miR-1260b, mediated by YY1, activates KIT signaling by targeting SOCS6 to regulate cell proliferation and apoptosis in NSCLC. Cell death \& disease 2019;10:112-.

30. Bucci B, Misiti S, Cannizzaro A, et al. Fractionated ionizing radiation exposure induces apoptosis through caspase- 3 activation and reactive oxygen species generation. Anticancer Res 2006;26:4549-57.

31. Cowan KJ, Storey KB. Mitogen-activated protein kinases: new signaling pathways functioning in cellular responses to environmental stress. J Exp Biol 2003;206:1107-15.

32. Eliopoulos AG, Young LS. Activation of the cJun $\mathrm{N}$-terminal kinase (JNK) pathway by the Epstein-Barr virus-encoded latent membrane protein 1 (LMP1). Oncogene 1998;16:1731-42.

33. Li L, Guo L, Tao Y, et al. Latent membrane protein 1 of Epstein-Barr virus regulates p53 phosphorylation through MAP kinases. Cancer Lett 2007;255:219-31.

34. Shklyaev SS, Namba H, Mitsutake N, et al. Transient activation of c-Jun NH2-terminal kinase by growth factors influences survival but not apoptosis of human thyrocytes. Thyroid 2001;11:629-36. 
Page 14 of 14

35. Tulalamba W, Janvilisri T. Nasopharyngeal carcinoma signaling pathway: an update on molecular biomarkers. Int J Cell Biol 2012;2012:594681.

36. Wang SS, Guan ZZ, Xiang YQ, et al. Significance

Cite this article as: Ma R, Gao P, Yang H, Hu J, Xiao JJ, Shi M, Zhao LN. Inhibition of cell proliferation and radioresistance by miR-383-5p through targeting RNA binding protein motif (RBM3) in nasopharyngeal carcinoma. Ann Transl Med 2021;9(2):123. doi: 10.21037/atm-20-6881
Ma et al. miR-383-5p inhibits NPC proliferation and radioresistance

of EGFR and p-ERK expression in nasopharyngeal carcinoma. Zhonghua Zhong Liu Za Zhi 2006;28:28-31.

(English Language Editor: K. Brown) 